\title{
Säulendhromatographische Trennung infektiöser und nicht-infektiöser Poliovirus-Nucleinsäure
}

\author{
Von G. Koch und H. Kubinski \\ Aus dem Laboratorium der Stiftung zur Erforschung der spinalen Kinderlähmung \\ und der Multiplen Sklerose (Prof. Dr. H. Pette), Hamburg-Eppendorf \\ (Z. Naturforschg. 17 b, 655-657 [1962] ; eingegangen am 9. Juli 1962)
}

\begin{abstract}
RNA isolated from highly purified poliovirus preparations consists of infectious and noninfectious fractions which can be separated by chromatography on methyl-esterified bovine serum albumin adsorbed on celite. The percentage of the infectious fraction varies from preparation to preparation.
\end{abstract}

Untersuchungen zur Charakterisierung der RNS, die aus gereinigten Polioviruspräparaten mit Hilfe der Phenolmethode gewonnen wurde, ergaben Hinweise, daß diese RNS heterogen ist. Holland und Mitarbb. ${ }^{1}$ und Drees und Koch ${ }^{2}$ konnten zeigen, $\mathrm{da} ß$ die Poliovirus-RNS in der analytischen Ultrazentrifuge nicht einheitlich sedimentiert. Die hierbei langsamer sedimentierende Fraktion ist sehr wahrscheinlich nicht infektiös.

Es erscheint wünschenswert, insbesondere auch für weitere Untersuchungen zur Charakterisierung der infektiösen RNS, die in den Präparaten - auf Grund der oben angeführten Beobachtungen - zu erwartende nicht-infektiöse von der infektiösen RNS zu trennen. Wir versuchten eine solche Trennung mit der von Mandell und Hershey ${ }^{3}$ angegebenen säulenchromatographischen Methode zu erreichen. Die Autoren hatten gezeigt, daß durch Chromatographie an methyliertem Serumalbumin, das an Kieselgel als Träger gebunden ist, Nucleinsäuren mit unterschiedlichem Molekulargewicht getrennt werden können. Mit Hilfe dieser von uns modifizierten Methode ${ }^{4}$ gelang uns eine Fraktionierung der aus infizierten Gewebekulturzellen isolierten RNS ${ }^{5}$. Die infektiöse Virus-RNS konnte weitgehend von den Fraktionen zellulärer RNS getrennt werden. Die Methode haben wir nun benutzt zur Analyse der RNS, die aus gereinigten Viruspräparaten isoliert wurde. Über die dabei gewonnenen Ergebnisse wird hier berichtet.

\section{Material und Methode}

Für die Untersuchungen wurde Poliovirus Typ I, Stamm Mahoney, verwendet. Als Wirtszellen dienten

1 J. J. Holland, B. H. Hoyer, L. C. McLaren u. J. T. SyverToN, J. exp. Medicine 112, 821 [1954].

2 O. Drees u. G. Косн, in Vorbereitung.

3 J. D. Mandell u. A. D. Hershey, Analyt. Biochem. [New York] 1, 66 [1960].
Zellen des permanenten Amnion-Zellstammes von Fernandes. Einzelheiten über Gewebekultur, biologischen Test, Virus- und RNS-Präparation sind einer vorausgegangenen Arbeit zu entnehmen ${ }^{6}$. Die chromatographische Analyse der RNS wurde unmittelbar im Anschluß an die Präparierung nach den Angaben von Mandell und Hershey ${ }^{3}$ und Kubinski und Mitarbb. ${ }^{4}$ durchgeführt. Die Auswertung der Analysen erfolgte wie dort bereits beschrieben.

\section{Ergebnisse}

Sechs ${ }^{32} \mathrm{P}$-markierte Viruspräparate wurden zur chromatographischen Analyse eingesetzt. Die Präparate wurden in größeren zeitlichen Abständen jedoch soweit wie möglich - unter gleichen Bedingungen hergestellt. Die chromatographische Analyse zeigt erhebliche Unterschiede in der Zusammensetzung der einzelnen Präparate. Ein von RNS-Präparat zu RNS-Präparat unterschiedlich großer Anteil der RNS wird - unter den von uns gewählten Bedingungen - in 0,65-m. NaCl-Lösung nicht an das Säulenmaterial adsorbiert. Der Anteil an nichtadsorbierter ${ }^{32} \mathrm{P}$-RNS liegt in der Regel zwischen 4 und $21 \%$ (Tab. 1). Bei zwei Experimenten allerdings ist er einmal kleiner als $1 \%$ und zum anderen um 62 Prozent. Aus chromatographischen Analysen der RNS infizierter Gewebekulturzellen ${ }^{4}$ entnahmen wir, daß solche RNS eine Sedimentationskonstante kleiner als $8 s$ hat. Wie zu erwarten, zeigt diese RNS-Fraktion im Plaquetest keine Infektiosität.

Die adsorbierte RNS - in der Regel also der Hauptanteil - wird in einem eng begrenzten Salzbereich von der Säule eluiert. ${ }^{32} \mathrm{P}$-Gehalt, optische

${ }^{4}$ H. Kubinski, G. Koch u. O. Drees, Biochim. biophysica Acta [Amsterdam] 61, 321 [1962].

5 H. Kubinski u. G. Koch, Virology 17, 219 [1962].

${ }^{6}$ G. Косн, Z. Naturforschg. 15 b, 656 [1960]. 
Dichte und Infektiosität der eluierten RNS sind einander proportional (Abb. 1 a). In drei Präparaten ist allerdings der zuerst eluierte RNS-Anteil nicht infektiös, zeigt aber noch eine Proportionalität zwischen optischer Dichte und ${ }^{32} \mathrm{P}$-Gehalt (Abb. $1 \mathrm{~b}$ und Tab. 1).

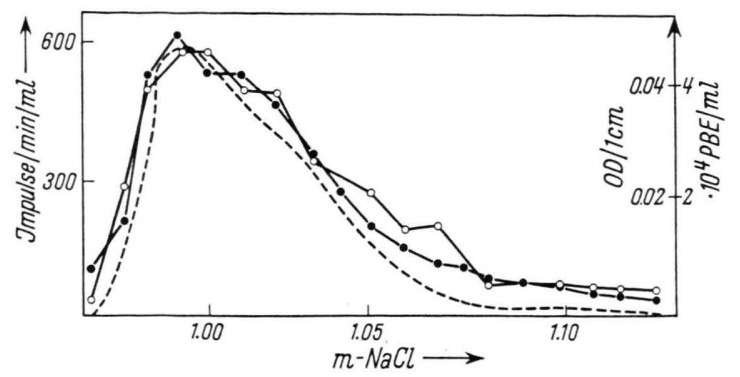

Abb. 1 a.

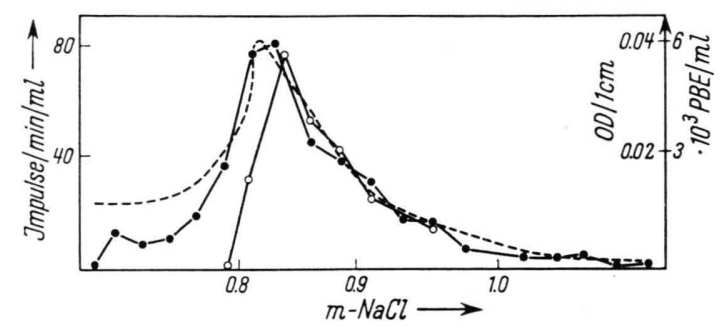

Abb. 1 b.

Abb. 1. Chromatographische Verteilung der adsorbierten RNS aus zwei gereinigten Viruspräparaten. - - - UV-Absorption, - - - - ${ }^{32} \mathrm{P}$-Gehalt, $-\mathrm{O}-\mathrm{O}-$ Plaque bildende Einheiten.

\begin{tabular}{|c|r|c|c|}
\hline \multirow{2}{*}{ Experiment } & \multicolumn{3}{|c|}{ RNS in \% } \\
\cline { 2 - 4 } & $\begin{array}{c}\text { nicht } \\
\text { adsorbiert } \\
\text { nicht } \\
\text { infektiös }\end{array}$ & $\begin{array}{c}\text { adsorbiert } \\
\text { nicht } \\
\text { infektiös }\end{array}$ & infektiös \\
\hline 1 & 19 & 0 & 81 \\
2 & 1 & 0 & 99 \\
3 & 21 & 0 & 79 \\
4 & 62 & 13 & 25 \\
5 & 11 & 7 & 82 \\
6 & 8 & 27 & 64 \\
\hline
\end{tabular}

Tab. 1. Anteil an infektiöser und nicht-infektiöser RNS in verschiedenen Viruspräparaten.

Ein kleiner Teil der RNS verbleibt an der Säule, er wird auch bei Salzkonzentrationen bis zu 1,2-m. nicht eluiert. Die Bestimmung der ${ }^{32} \mathrm{P}$-Aktivität einzelner Säulenabschnitte hat ergeben, daß der kleinere Anteil dieser zunächst nicht eluierten RNS irre- versibel an das Papierpulver gebunden wird, das die Säulenbasis bildet, und der größere Anteil der primär nicht eluierten RNS vom Säulenmaterial selbst reversibel zurückgehalten wird. Diese letztere Fraktion kann auch durch eine folgende Gradientenelution teilweise noch von der Säule zurückgewonnen werden. Eine ergiebigere Elution wird erreicht, wenn die Säule zu einer zweiten Nucleinsäure Chromatographie benutzt wird. Eine solche Rückgewinnung der RNS von der Säule wird in gleichem Ausmaß durch Chromatographie von nieder- und hochmolekularer RNS - aus infizierten oder aus nicht-infizierten Gewebekulturen - und auch durch Chromatographie von DNS erreicht.

Die während der ersten Chromatographie nicht eluierte ${ }^{32} \mathrm{P}$-RNS verläßt die Säule dann bei 2 Salzkonzentrationen und zwar: a) bei einer Konzentration von $0,9-1,2-m$., die charakteristisch ist für die Elution der Virus-RNS und b) bei der Konzentration, während der der Hauptanteil der zur zweiten Chromatographie benutzten Nucleinsäure die Säule verläßt.

Durch Bestimmung der ${ }^{32} \mathrm{P}$-Aktivität und durch Infektiositäts-Titration konnten wir zeigen, daß die nun eluierte ${ }^{32} \mathrm{P}$-RNS in beiden Fraktionen weitgehend aus hochmolekularer infektiöser RNS besteht. Aus anderen Untersuchungen entnehmen wir, daß der Anteil an primär nicht-eluierter RNS um so größer ist, je höher das Molekulargewicht der RNS ist. Primär nicht eluierte infektiöse RNS kann demnach von der Säule durch verschiedene Nucleinsäuren verdrängt werden, weitgehend unabhängig von ihrer chemischen und physikalischen Zusammensetzung.

\section{Diskussion}

Verschiedene Autoren ${ }^{3,4,7,8}$ konnten zeigen, daß durch Chromatographie an methyliertem Serumalbumin Nucleinsäuren nach ihrem Molekulargewicht getrennt werden können. Die aus gereinigten Polioviruspräparaten isolierte RNS wird dabei in 2-3 Fraktionen getrennt, von denen nur eine, die hochmolekulare, infektiös ist. Im allgemeinen wird der größte Anteil der RNS in dieser infektiösen Fraktion gefunden. Daraus folgt, daß die auf die Anzahl physikalischer Teilchen bezogene niedere

7 C. Cocito, A. Prinzie u. P. De Somer, Nature [London] 191, 573 [1961].

8 L. Philipson, J. gen. Physiol. 44, 899 [1961]. 
Infektiosität von intakten Polioviren nicht durch das Vorliegen einer inkompletten RNS in einem hohen Prozentsatz der Teilchen bedingt ist.

Die relativ niedere Infektiosität der RNS wird nicht durch eine teilweise Spaltung der RNS bei dem Isolierungsverfahren hervorgerufen. Damit wird die schon früher gemachte Annahme ${ }^{9}$ bestätigt, daß die RNS bei der Isolierung mit der Phenolmethode nicht wesentlich in ihrer Struktur und biologischen Aktivität verändert wird. Gleichzeitig wird die Hypothese erhärtet, daß die geringe Empfänglichkeit der Zellen für die niedere Infektiosität der RNS verantwortlich zu machen ist.

Die Virus-RNS-Präparate enthalten verschieden hohe Anteile an hochmolekularer RNS. Eine direkte Beziehung zwischen dem Anteil an hochmolekularer RNS und der Infektiosität des Virus-Ausgangsmaterials konnten wir nicht finden. $\mathrm{Da} \beta$ eine Heterogenität der Virus-RNS durch eine Anwesenheit von zellulärer RNS in den gereinigten Viruspräparaten bedingt wird, ist wenig wahrscheinlich, da der Hauptanteil der zellulären RNS bei anderen Salzkonzentrationen die Säule verläßt und die zelluläre RNSFraktion, die kurz vor der Virus-RNS im Eluat er-

${ }^{9}$ G. Kосн, Zbl. Bakteriol. Parasitenkunde, Infektionskrankh. Hyg., Abt. I, Orig. 184, 165 [1962]. scheint, in vitro sehr unstabil ist ${ }^{11}$ und deshalb zu erwarten ist, daß sie bei der Viruspräparation abgebaut wird. Worauf die unterschiedliche Zusammensetzung der einzelnen RNS-Präparate (Tab. 1) zurückzuführen ist, bleibt allerdings noch ungeklärt.

In diesem Zusammenhang erscheinen Untersuchungen von Drees ${ }^{10}$ erwähnenswert, die eine von Viruspräparat zu Viruspräparat unterschiedliche Stabilität der Viren aufzeigen. So werden in einigen Viruspräparaten bis zu $90 \%$ der Viren innerhalb von 6 Tagen bei $37^{\circ} \mathrm{C}$ in Protein und RNS gespalten, während in anderen Viruspräparaten die meisten Teilchen unter gleichen Bedingungen physikalisch intakt bleiben.

Weitere Untersuchungen sind nötig, um die Ursachen für die unterschiedliche Zusammensetzung der Viren in einzelnen Viruspräparaten zu klären.

Herrn Professor Honst danken wir für wertvolle Hilfe bei der Verwendung von ${ }^{32} \mathrm{P}$.

Die Arbeit wurde durchgeführt mit Unterstützung der Deutschen Forschungsgemeinschaft und des Vereins zur Förderung und Bekämpfung der spinalen Kinderläh m ung e. V., Bielefeld.

10 O. DreEs, unveröffentlicht.

11 H. Kubinski u. G. Косн, J. molecular Biol., im Druck. 